v1kwomer@ed.ac.uk

Cite this as: BMJ 2021;372:n4 http://dx.doi.org/10.1136/bmj.n4 Published: 05 January 2021

\section{Why were breastfeeding women in the UK denied the covid-19 vaccine?}

\section{Absent data are not a valid justification for exclusion}

Helen Hare, Kate Womersley

On 30 December, a month after licensing the Pfizer BioNTech covid-19 vaccine, the UK's Medicines and Healthcare Products Regulatory Agency (MHRA) revised its guidance to enable pregnant and breastfeeding women to receive it. The change came after pressure from campaigners, clinicians, and affected women, including a piece we wrote for $B M J$ Opinion. The MHRA previously recommended that breastfeeding women be denied the vaccine, which was interpreted by NHS trusts as a blanket ban. This was at odds with the EU, US, and Canada, where women have been encouraged to make risk-benefit decisions based on their own circumstances. The MHRA's U turn is welcome news, but why were breastfeeding women initially excluded?

If the MHRA held public advisory committee meetings like the US when assessing vaccines for emergency use applications, the rationale for their original and revised recommendations could be openly scrutinised. ${ }^{1}$ The MHRA's initial position, which disregarded a breastfeeding woman's level of exposure to the virus or her likelihood of developing a severe form of the disease, seemed to result from a lack of evidence. Pfizer, AstraZeneca, and Moderna vaccine trials did not enrol breastfeeding women and so no specific safety data are currently available. But absent data are rarely a valid justification for broad exclusions. Men who are trying to conceive have not been cautioned about the vaccine, even though no data exist about possible effects on spermatogenesis. Any data gap leaves open a possibility of risk. No plausible biological mechanism for how an inactivated, recombinant vaccine would cause harm to a breastfed baby has yet been proposed. Theoretical risk must be weighed against the established benefits of acquiring immunity to covid-19 and of continued breastfeeding.

The "green book," which provides information on vaccines in the UK, did not offer further explanation of the MHRA's stance, merely stating that the licensor "advised vaccination should not be given while breastfeeding." 2 The green book saw this as a "very precautionary approach" which protected women and infants. But, in our view, the MHRA's approach was not cautious: it was reckless to insist on exposing these women, their families, and-in the case of healthcare workers-their patients, to a potentially lethal virus. It also threatened the UK's already low breastfeeding rates.

The US approach has been quite different. Recommendations from the Advisory Committee on Immunization Practices, approved by the Centers for Disease Control, agreed from the outset that breastfeeding women could choose to receive or refuse the Pfizer BioNTech vaccine. The American College of Obstetricians and Gynecologists released a practice advisory stating "vaccines should not be withheld from lactating individuals who otherwise meet criteria for vaccination.” The Royal College of Obstetricians and Gynaecologists in the UK made no such statement supporting a woman's right to choose, tweeting that "there isn't sufficient evidence to recommend the routine use of covid-19 vaccines to pregnant or breastfeeding women."3

The assumption made by the MHRA seemed to be that the number of doctors, nurses, and carers at work who are breastfeeding is vanishingly small. That several thousand healthcare workers are breastfeeding while caring for patients may come as a surprise to many-although it shouldn't. Guidelines from the NHS and the World Health Organization strongly support breastfeeding until an infant is two years of age and beyond. These targets are often thought relevant only to women in developing countries, and it's wrongly presumed that for mothers in the West, breastfeeding ceases to be of benefit after six months. No wonder breastfeeding rates in the UK are some of the lowest in the world, with 8 out of 10 women stopping before they want to. 45

Any woman should be enabled to continue breastfeeding for reasons of maternal and infant comfort, health promotion, or medical necessity. To support breastfeeding beyond the first few months is a public health priority which policy makers cannot afford to ignore. The MHRA's previous guidance reinforced society's perception of breastfeeding as a lifestyle choice. This belied the effort and expense incurred by those who breastfeed, a physically and emotionally demanding commitment which does not come naturally to many infants or their mothers. Many women cite the importance of breastfeeding for their mental health, and as a powerful way to reconnect with their baby after a day's separation. When women faced a decision to either stop breastfeeding or not be vaccinated, many felt personally and professionally conflicted. Stopping feeding abruptly is not like cancelling a subscription: it is associated with negative emotions-known as breastfeeding grief-risk of engorgement, and mastitis, as well as being a sudden physical and emotional transition for a child. Doctors told us they were prepared to lie in order to get the vaccine, even though this was in breach of their duty to act with

The MHRA now recommends breastfeeding women should have a "risk-benefit discussion with a healthcare provider" before receiving a covid-19 vaccine. While a step in the right direction, this is not honesty and integrity. 
a solution. There is currently minimal capacity in an overwhelmed NHS for appointments to discuss a decision for which there is no data. When consenting at the point of being vaccinated, the Joint Committee on Vaccination and Immunisations states that a breastfeeding patient "should be informed about the absence of safety data for the vaccine in breastfeeding women." 6 This is not a discussion, it is a washing of hands. We have also heard from breastfeeding doctors who are still being denied the vaccine, despite the new regulations. Even when the patient group direction and trust specific paperwork is updated, lasting damage has been done to the confidence of breastfeeding women and those administering the vaccines.

Allowing those who breastfeed to receive a covid-19 vaccine should not set a precedent for condoning gaps in evidence. Women must be allowed to make choices about their bodies, but this is a travesty of empowerment if female populations are not also studied. There are understandable ethical difficulties in trialling novel therapies in lactating women, but robust research on the vaccines' safety in breastfeeding must nevertheless be an urgent priority. Currently no European trials are underway in humans to collect such data, even though many breastfeeding healthcare workers are willing to contribute. On one UK based Facebook group alone, over 400 breastfeeding doctors stated their enthusiasm to get vaccinated, be followed up, and then donate milk samples for analysis.

This data gap is not an anomaly. It is the result of a system of researching and licensing drugs that routinely discriminates against women, excludes them from the evidence base, and denies them the right to make informed choices about their own health. We implore researchers, industry leaders, and the MHRA to remember that pregnant and breastfeeding women are essential patient populations, not merely women who can wait.

$\mathrm{HH}$ is breastfeeding an 18 month old, KW is breastfeeding a 9 month old.

No competing interests.

Not commissioned, not peer reviewed.

1 US Food and Drug Administration. Common questions and answers about FDA advisory committee meetings. 2018. www.fda.gov/advisory-committees/about-advisory-committees/commonquestions-and-answers-about-fda-advisory-committee-meetings.

2 Covid-19 green book: chapter 14a. December 2020. https://assets.publishing.service.gov.uk/government/uploads/system/uploads/attachment_data/file/943663/Greenbook_chapter_14a_v3.pdf.

3 RoyalCollegeObsGyn. 4 December 2020. https://twitter.com/RCObsGyn/status/1334849583877386240.

4 Unicef. Breastfeeding in the UK. www.unicef.org.uk/babyfriendly/about/breastfeeding-in-the-uk.

5 Association of Breastfeeding Mothers. Breastfeeding beyond infancy: a guide for GPs. https://abm.me.uk/wp-content/uploads/GP-Guide-Breastfeeding-beyond-infancy.pdf.

6 Joint Committee on Vaccination and Immunisation. Advice on priority groups for covid-19 vaccination. 30 December 2020. https://assets.publishing.service.gov.uk/government/uploads/system/uploads/attachment_data/file/948338/jcvi-advice-on-priority-groups-for-covid-19-vaccination30-dec-2020.pdf. 